\title{
Análisis de estrategia y plan comunicacional: Campaña contra el Cáncer de Mama de la FALP
}

\author{
doi: $10.33264 /$ rpa.201901-01
}

Cielo Yaneth Lengua Buelvas

Valeska Dahiana Fajardo Henríquez

Guillermo Antonio Gamboa Peña

\section{Resumen}

Para Porter (2015), la estrategia en las organizaciones es una combinación de fines que busca la compañía y las políticas con que trata de alcanzarlos. El objetivo de este estudio es analizar la estrategia y plan de comunicación de una campaña comunicacional enfocada a la concientización, que en este caso, corresponde a la campaña contra el Cáncer de Mama que realiza la Fundación Arturo López Pérez (FALP). Por medio de entrevistas semiestructuradas a la Gerencia de Marketing y Comunicaciones de la fundación, además de expertos nacionales e internacionales en el área, se encontró que aunque la institución de salud enfoca sus campañas en función de su estrategia corporativa, aún falta por reforzar aspectos en identidad y cultura organizacional que potencian la base de una campaña comunicacional.

Palabras clave: estrategia, comunicación, comunicación estratégica, campañas comunicacionales, concientización.

\section{Abstract}

For Porter (2015), the strategy in organizations is a combination of goals that the company seeks and the policies with which it tries to achieve them. The objective of this study is to analyze the strategy and communication plan of a communication campaign focused on awareness, which in this case, corresponds to the campaign against breast cancer carried out by the Arturo López Pérez Foundation (FALP); to know what are the characteristics of the campaign to raise awareness among the population about breast cancer?. Through semi-structured interviews to the Marketing and Communications Management of the foundation, in addition to national and international experts in the area, it was found that although the health institution focuses its campaigns based on its corporate strategy, there is still a need to strengthen aspects in identity and organizational culture that strengthen the base of a communicational campaign that seeks to position a topic in the minds of people. The analysis of the research allows to conclude the FALP campaign complies with the main features, but has shortcomings in the process of measurement and evaluation that allows to determine the impact of the campaign on the target population. 
Keywords: strategy, communication, strategic communication, communication campaigns, organizational identity, awareness, Chile.

\section{Introducción}

Las campañas comunicacionales y publicitarias cada vez están creciendo más en número y a la vez, el elemento "evaluación de la eficacia de la publicidad" se está tornando determinante (Águeda, 2008) ya que se encarga de definir si el trabajo realizado y los recursos invertidos fueron efectivos y tuvieron los resultados que se esperaban.

Actualmente, aún existen empresas que no miden los efectos de sus campañas para efectuar mejoras, solo repiten año a año las mismas acciones, pero cuando hablamos de una temática de interés mundial como el cáncer de mama, de un problema que afecta a millones de mujeres, de las cuales año a año mueren más de 458.000 en el planeta por esta enfermedad (IARC Globocan, 2008) y en Chile, por ejemplo, 1.422 personas fallecieron en 2014 (Ministerio de Salud de Chile, 2017). Se entiende que al hacer campañas utilizando esta temática en particular, tiene sin duda una trascendencia y un interés total en concientizar a la población, por tanto se deben evaluar constantemente las acciones que se están efectuando directamente con la comunidad para ver sus efectos y determinar si realmente se está logrando impactar.

La Fundación Arturo López Pérez - FALP, es la institución líder en el país en los tratamientos contra los diversos cánceres, teniendo la más alta tecnología para atender a sus pacientes provenientes de diversos rincones de Chile. Esta institución, realiza todos los años en octubre una campaña enfocada al cáncer de mama, con el objetivo de concientizar y enseñar a la población, tanto hombres y mujeres, sobre cómo prevenir este cáncer.

Teniendo en cuenta las ideas planteadas anteriormente como: la relevancia del tema "cáncer de mama" a nivel mundial y local, la importancia de la concientización, lo primordial que es evaluar una campaña comunicacional o publicitaria, sobre todo cuando son temas tan reales y que impactan a millones de personas, y el papel fundamental que juega la FALP como institución líder en los tratamientos contra los diversos cánceres en Chile, los investigadores se plantean lo siguiente:

1. ¿Cuáles son las características de la campaña para lograr concientizar a la población respecto al cáncer de mama?

2. ¿Cómo se relaciona la campaña del cáncer de mama con la estrategia comunicacional de la institución? 
3. ¿Cuáles son los aspectos importantes de las evaluaciones de eficacia luego de implementarse la campaña? En caso que sean realizadas por parte de la FALP.

4. ¿Existirán otras herramientas o acciones que potencien la campaña comunicacional de la FALP respecto al cáncer de mama y mejoren sus resultados y alcances?

Las preguntas que plantean los investigadores, se enfocan en analizar principalmente el periodo de implementación de una campaña comunicacional con esta relevancia, en la evaluación que realizan luego de pasado el mes de octubre y sus resultados, en analizar la estrategia de la institución y si se considerarían nuevas tácticas, acciones o cambios en la campaña comunicacional contra el cáncer de mama para potenciarla, si ese fuera el caso.

\section{Hipótesis}

Una campaña comunicacional que busca concientizar a su público objetivo, es efectiva cuando su planeación y estructura están articuladas a la estrategia global de la institución, junto con enfocarla en las características sociales de las personas a las que va dirigida, además de realizar las mediciones de efectividad correspondientes al momento de aplicada la campaña comunicacional.

\section{Marco teórico}

El desarrollo y análisis de autores expuestos en el marco teórico, ayudarán de gran manera a los investigadores a poder indagar de una manera más completa, la campaña comunicacional que la FALP realiza en el mes de octubre, relacionada a cáncer de mama.

\section{Comunicación}

La comunicación es una necesidad inherente al ser humano y una herramienta fundamental e indispensable en la sociedad y su estructuración. Este proceso innato en los seres humanos permite la interacción con los demás, generando un intercambio y un compartir informativo, de conocimientos y de experiencias.

En su concepto técnico la comunicación es: "un proceso más o menos complejo en el que dos o más personas se relacionan, a través de un intercambio de mensajes con códigos similares" (Román, 2005). Es un proceso que permite el intercambio de información y la construcción social de los individuos, logran transmitir y recibir información de todo tipo de índole. 


\section{Comunicación y estrategia:}

En la actualidad, y en un concepto más especializado la comunicación es considerado un proceso que permite a entidades crear estrategias encaminadas a comunicarse de manera acertada con los públicos objetivos, para persuadir con un mensaje claro. (Jaramillo, 2011, pág. 4): “Una estrategia es el proceso seleccionado a través del cual se prevé alcanzar un resultado, es decir, un conjunto de actividades, fines y recursos que se analizan, organizan y plantean de tal manera que cumplan con los objetivos que el proyecto plantea".

La comunicación estratégica es un conjunto de acciones, tácticas, las cuales aplicadas al público objetivo logran informar y persuadir a la audiencia. "Is coordinating the things you do and say in support in your objectives. More formally, I define strategic communication as coordinated actions, messages, images, and other forms of signaling or engagement intended to inform, influence, or persuade selected audiences in support of national objectives". (Paul, 2011).

La comunicación estratégica se explica que es global, debe integrar las partes de la organización como un todo y gestionarla de tal manera que traiga beneficios y aprendizajes a la organización.

\section{Estrategia:}

Actualmente, al hablar de estrategia se puede referenciar la nueva teoría estratégica para entender las innovadoras miradas en el aspecto comunicacional. (Herrera Echenique, 2017) "La NTE propone trabajar con los paradigmas actuales de la ciencia y no con los del siglo XVII. Y frente a una visión de la realidad dual, fragmentaria y estática, nos aporta una visión multidimensional, compleja, fluida y, a veces caótica. La nueva teoría estratégica aspira a proporcionar reglas hermenéuticas y articuladoras".

La comunicación estratégica debe estar integrada de manera transversal en la estrategia global de la compañía, para que todas las acciones en términos de comunicación estén enfocadas en un mismo sentido y logren responder a los lineamientos y políticas de la organización. 


\section{Comunicación y Salud:}

Teniendo claridad de qué es la comunicación estratégica, es necesario ahora establecer una relación entre comunicación y salud, que permite entender la importancia de la campaña adelantada por la FALP.

Como bien lo indica (Alcalay, 1992) “La importancia de la comunicación en el ámbito de la salud es clara. Existe una disparidad entre los avances logrados por la medicina y el conocimiento y la aplicación de estos por el público. Mientras que los profesionales de la salud tienen grandes conocimientos sobre la prevención de las enfermedades y la promoción de la salud, no saben necesariamente cómo comunicar efectivamente esa información tan vital para la sociedad".

En temas de salud, los profesionales de la comunicación cumplen un papel fundamental en lograr generar estrategias que trabajen en pro de la prevención de enfermedades e involucren a profesionales de la salud con los públicos objetivos.

\section{Campañas Cáncer de Mama:}

Luego de conocer las bases teóricas de la comunicación, es pertinente relacionar con el tema de campañas de cáncer de mama, donde se encuentran antecedentes importantes, es el caso de una investigación realizada en Bucaramanga, Colombia, denominada: (Toquica am, 2015) "Estrategias de comunicación para vincular a la comunidad a programas de detección temprana de cáncer de mama”, este estudio de análisis tuvo como objeto reconocer diferentes estrategias de comunicación masiva implementadas para la vinculación de la comunidad a los programas de detección temprana de cáncer de mama.

El cáncer de mama es una problemática de salud pública que a nivel mundial se trabaja en lograr disminuir el índice de mortalidad e informar a la población de la importancia de la detección temprana, (Santos Da Silva, 2014) indican que: "El cáncer de mama es una enfermedad multifactorial en la que los factores genéticos y ambientales contribuyen a su aparición".

Según las estadísticas de mortalidad proporcionadas por el Departamento de Estadísticas e Información en salud (DEIS) del Ministerio de Salud, en el Chile, en el año 2008, el cáncer de mama alcanzó una tasa de mortalidad de 14,5 por 100.000 mujeres, ocupando el segundo lugar de la mortalidad por cáncer. Las comunas con mayor riesgo de mortalidad por cáncer de mama en mujeres se ubican en la Región Metropolitana, la Región de Valparaíso y la Región de Magallanes. En el año 2013 se 
registraron 1.389 muertes por cáncer de mama en mujeres, correspondiente a 11,8\% de defunciones oncológicas femeninas. (Gloria Icaza, 2015).

\section{Concientización}

Uno de los principales objetivos de compañas comunicacionales que buscan educar e insertar un tema sensible en la población es lograr concientizar a la comunidad y su público objetivo. Se entiende por concientización "como un proceso de evolución continua tendiente hacia un único propósito: el de generar praxis. De igual manera podemos concluir que no toda unión entre actividad teórica y actividad práctica puede considerarse como praxis, sólo podrá serlo cuando apunte, de manera intencionada, a afectar la estructura social". (Cerullo, Renato, and Esther Weisenfeld, 2005).

Para el pedagogo brasileño Paulo Freire, concientizar es un “... proceso educativo mediante el cual las personas y los grupos sociales toman conciencia crítica del mundo histórico-cultural en que viven y asumen las responsabilidades y emprenden las acciones necesarias para transformarlo". (Freire, 1999).

\section{Metodología}

La institución seleccionada para la investigación es la FALP, en la cual se realizó un análisis de comunicación estratégica sobre la campaña anual del cáncer de mama, realizada en el mes de octubre.

El Instituto Oncológico es un centro de referencia en cáncer, dedicado al diagnóstico, tratamiento, investigación y docencia. Busca la calidad y excelencia a través del mejoramiento continuo, en un ambiente de respeto, calidez y profesionalismo para sus pacientes, sus familias y la comunidad.

La investigación es de tipo cualitativo, de carácter descriptivo, en la cual se realizaron entrevistas semiestructuradas y se consultó a un panel de expertos.

Las entrevistadas en la FALP fueron Bernardita Vittini de la Barrera, Gerente de Comunicaciones y Marketing y Paula Rojas Escobar, Jefe Comunicaciones Corporativas. Las preguntas estaban dirigidas a saber cómo realizan la campaña, sus enfoques, sus acciones, y cómo se apega a la estrategia de la institución. 
Las profesionales indican en las entrevistas que La FALP tiene una agenda que está alineada a un calendario de salud internacional. Todos los canceres tienen un día mundial de conmemoración, y la FALP aplica este calendario para sus campañas.

Parte del quehacer de la FALP tiene que ver con la investigación, la detección precoz, la prevención, el tratamiento y los cuidados paliativos cuando ya corresponden. Por tal razón las campañas están articuladas a la estrategia general.

En RRSS la FALP tiene presencia desde hace varios años, con un trabajo importante que se refleja en el número de seguidores, el cual en la actualidad es cercano a 13.000 seguidores, todo ha sido de manera orgánica y es solo el tema de cáncer. Con interacciones por parte de los seguidores que permite analizar la acogida de las campañas a nivel digital.

La FALP durante el mes de octubre realiza diversas actividades como parte de la campaña comunicacional. Una de las acciones principales es la caminata en convenio con AVON. Esta caminata busca recaudar fondos para donar mamografías gratuitas a mujeres de escasos recursos y de regiones alejadas, donde la FALP Ilega con un grupo de profesionales a realizar los exámenes.

El objetivo de la campaña es lograr concientizar a la población de la importancia de detectar el cáncer en su etapa inicial. La FALP cada año fija un propósito diferente como involucrar a familiares de pacientes con cáncer, concientizar a las mujeres que el cáncer puede presentarse a cualquier edad, persuadir a las mujeres de dedicar tiempo para realizar los controles.

\section{Investigación}

\section{Panel de Expertos}

Respecto al panel de expertos, los entrevistados fueron Roberto Arancibia, publicista e international speaker; Érika Benitez, comunicadora social, directora de proyectos sociales; Jorge Torres, experto en publicidad, director agencia de branding Polaris y asesor comunicacional; Astrid Gómez, comunicadora social doctora en educación, docente Universidad de Pamplona, Colombia; Catalina Camargo, comunicadora social, docente e investigadora, directora del programa de Comunicación Social Universidad de Pamplona, Colombia; Paula Sánchez, jefa de comunicaciones del Museo Histórico de la Memoria de Chile y jefa de programación; Elvis Fernando Ríos Pacheco, Comunicador Social, magister en Dirección del Desarrollo Loca, experto en Comunicación Corporativa, Conferencista y Ana Paola Balmaceda, Comunicadora 
Social, Magister en Educación, docente Universidad Francisco de Paula Santander Ocaña, Colombia. Las preguntas a los expertos estaban enfocadas a la importancia de la estrategia en las campañas, a saber la elaboración de éstas y la relevancia de plasmar la identidad en ellas.

Para la elaboración de las preguntas, se utilizaron las variables del Modelo MasterBrand de Joan Costa: Estrategia, Identidad y Cultura organizacional.

Las mayoría de los expertos del panel indican que el impacto de la campaña es una característica relevante para medir su efectividad. Se debe considerar dentro de la campaña una articulación entre mensaje y el medio, dos herramientas para tener en cuenta en el momento de diseñar la misma. Se indica, por parte de los expertos, que conocer la audiencia es una característica que aporta en gran medida a impactar en la conciencia de la población a la cual va dirigida la campaña.

Según lo indicado por los expertos, se debe tener claridad sobre el objetivo de la campaña, lo cual la FALP realiza de manera acertada y fija un propósito para este año.

Para los expertos la campaña debe estar articulada a la estrategia de la institución y alineada a los objetivos generales, lo que es útil para la investigación y permite identificar que la campaña, como lo indica uno de los expertos, no puede ser diseñada y ejecutada de manera aislada a la estrategia global de la institución.

Finalmente, el análisis del aporte de los expertos indica que es sumamente importante medir siempre el impacto de las campañas, la evaluación es tan importante con la misma campaña; porque permite la retroalimentación y la mejora continua en la aplicación de las acciones que conforman la campaña.

\section{Desarrollo}

\section{Identidad Corporativa}

Dentro de las variables del Modelo MasterBrand se encuentra en primer lugar la identidad de marca, la cual indica que la identidad institucional es aplicada en las campañas de comunicación para mantener unidad en la marca. En este variable la FALP realiza un trabajo adecuado que se refleja en la campaña de comunicación de lucha contra el cáncer de mama, donde aplicado los principios corporativos logra transmitir unidad en el uso de la imagen corporativa y evidencia una relación directa de la campaña con la estrategia de la fundación. 


\section{Cultura Organizacional}

La FALP hace una gestión de marca por medio de su cultura organizacional la cual está implícita en la campaña de lucha contra el cáncer de mama. La FALP es considerada por los stakeholders como una fundación que lucha contra el cáncer, y esto permite inferir que la marca realiza un proceso comunicativo acertado, a toda vez, que logra transmitir sus políticas y valores a los públicos objetivos.

La cultura organizacional de la FALP estipula la forma como funciona la fundación y esta se refleja a través de su estrategia general. La fundación esta formada por valores y normas definidas que permite a cada uno de los individuos identificarse con ellos. Esta cultura organizacional favorece el sentido de pertenencia con la FALP, por ello en la campaña se ven reflejados los principios de misión y valores de la fundación.

\section{Estrategia Corporativa}

La estrategia de la FALP es la de posicionarse como la institución más importante en la lucha contra el cáncer en Chile. Actualmente, la Falp es el principal referente de lucha contra el cáncer en el país, lo que permite inferir que la estrategia corporativa esta siendo ejecutada de manera correcta, sin embargo, la fundación sigue trabajando en fortalecer su estrategia corporativa para aumentar la reputación de marca.

En el mandato funcional de la Falp se considera prevenir y combatir el cáncer, y este aspecto la comunicación cumple un papel primordial para relacionarse asertivamente con los públicos objetivos.

\section{Análisis Panel de Experto}

Respecto a lo que los investigadores pudieron rescatar de las diversas entrevistas con los expertos que componen el panel, dos de las tres variables del Modelo MasterBrand, se destacan más, éstas son Identidad y Estrategia.

Estas dos variables, se presentan como aspectos a integrar en la campaña desde el día uno de su planeación. Son la base de la campaña ya que desde allí se comienzan a idear las directrices a seguir, por tanto que la campaña integre la estrategia organizacional y que refleje aspectos como el quehacer institucional, los valores, su misión y visión, es algo obligatorio en cómo debe componerse una campaña. 
Finalmente, el análisis del aporte de los expertos indica que es sumamente importante medir siempre el impacto de las campañas, la evaluación es tan importante con la misma campaña; porque permite la retroalimentación y la mejora continua en la aplicación de las acciones que conforman la campaña.

\section{Campaña contra el Cáncer de Mama de la FALP}

La campaña comunicacional contra el Cáncer de Mama que realiza la Fundación Arturo López Pérez todos los años, se desarrolla en octubre ya que, en este mes, se conmemora el Día Mundial Contra el Cáncer de Mama, específicamente el día 19.

Debido a esto, la Gerencia de Marketing y Comunicaciones de la institución, realiza una serie de acciones, la cuales se comienzan a planear desde el mes de abril-mayo y que tienen año a año un mensaje y enfoque distinto. Esto se decide en base a la experiencia y contacto con los pacientes que tienen los doctores de la fundación; desde allí se desprende el eje central de la campaña, de allí nace el slogan y acciones, las que son alineadas con el quehacer de la institución.

En lo que respecta al 2018, el slogan fue "No importa la edad, importa que te cuides", por lo que las tácticas y acciones fueron enfocadas a transmitir y reforzar ese mensaje. En la gráfica que se presenta a continuación y que fue difundida en diversas plataformas, se muestra a una mujer adulta y a una más joven, dando a entender que las mujeres de todas las edades deben preocuparse del cáncer de mama.

La campaña comunicacional de la Falp se puede resumir de la siguiente forma:

Figura 1: Campaña Contra el Cáncer de Mama de la FALP 2018.

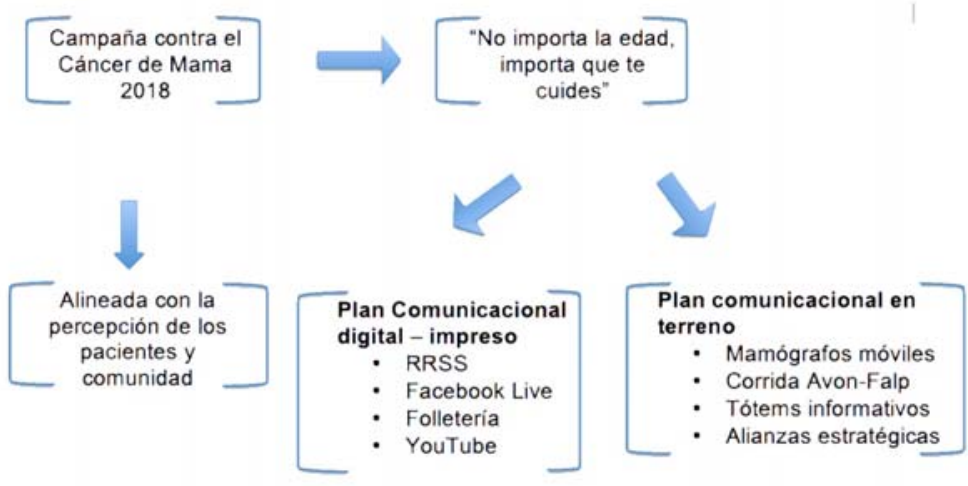

Fuente: elaboración propia. 
A continuación, se pasan a detallar los dos planes comunicacionales que plantea la Falp:

\section{Plan Comunicacional Digital - Impreso}

RRSS: a través de publicaciones tanto en Instagram como en Facebook, se difunde el mensaje de que octubre es el mes de la lucha contra el cáncer de mama, consejos de prevención y educación respecto a la enfermedad a través de comunicados y gráficas.

Facebook Live: Como una instancia de comunicación directa con los pacientes y la comunidad, se realizó durante octubre un Facebook Live a cargo del cirujano oncólogo Dr. Badir Chahuán, quien respondió inquietudes sobre el cáncer de mama, enfermedad que representa la primera causa de muerte oncológica en las mujeres chilenas. El video obtuvo 2.200 reproducciones y 104 comentarios.

Folletería: diseño de dos tipos de folletería para distribuir en las diferentes actividades: la primera incluye los pasos del auto examen con el objetivo de detectar a tiempo el cáncer de mama y la segunda, explica los factores de riesgo generales y hereditarios que podrían ser la causa de la enfermedad.

YouTube: la FALP posee un canal en YouTube con 313 suscriptores y 66 videos. Dicha plataforma se utiliza para subir entrevistas en importantes canales nacionales de sus doctores, además de testimonios de pacientes y spots. Relacionado a la campaña contra el cáncer de mama, se subió un video promocional de la caminata corrida Avon-Falp en el mes de agosto, video que obtuvo 794 reproducciones.

\section{Plan Comunicacional en Terreno}

Mamógrafos móviles que recorren el país: si bien esta es una acción que se extiende a lo largo del año, se intensifica con mayor fuerza en octubre. Se trata de 6 mamógrafos móviles con tecnología de punta que buscan llegar a zonas aisladas y de difícil acceso, donde las mujeres tienen dificultades de traslado para efectuar sus mamografías. Estas clínicas móviles benefician alrededor de 60 mil mujeres anuales a lo largo del territorio nacional.

Corrida Caminata Avon-Falp: ésta es una de las acciones más importantes de la campaña. En la versión número 14, participaron más de 9 mil personas y se llevó a cabo el 7 de octubre en Parque Balmaceda. En la oportunidad, además de la corridacaminata, se realizan activaciones relacionadas a realizarse el auto examen y entrega 
de folletería educativa. En la actividad también participaron rostros televisivos y autoridades de gobierno, con el fin de incluir a todos los actores de la comunidad.

Redes sociales: a través de publicaciones en Facebook e Instagram, se invita a participar e inscribirse en la iniciativa. Se difunde con el slogan "Para ganarle al Cáncer de Mama”.

Página web institucional y de la corrida: a través de la página www.instituciononcologicafalp.cl y www.corridaavon.cl se realiza la difusión y convocatoria a la actividad. En ellas se publican notas de prensa, gráficas, inscripción, preguntas frecuentes, entre otras.

Tótems informativos: a través de diversas alianzas con instituciones privadas, se instalaron puntos informativos con el apoyo de tótems que enseñaban la realización del autoexamen, entrega de volantes y chapitas con el lazo rosa, con el fin de concientizar a la comunidad sobre la importancia del autoexamen como prevención de esta enfermedad. Dichos puntos se instalaron en Metro Baquedano, Mall Alto Las Condes y en el hall de la Falp.

Imagen 18, 19 y 20:

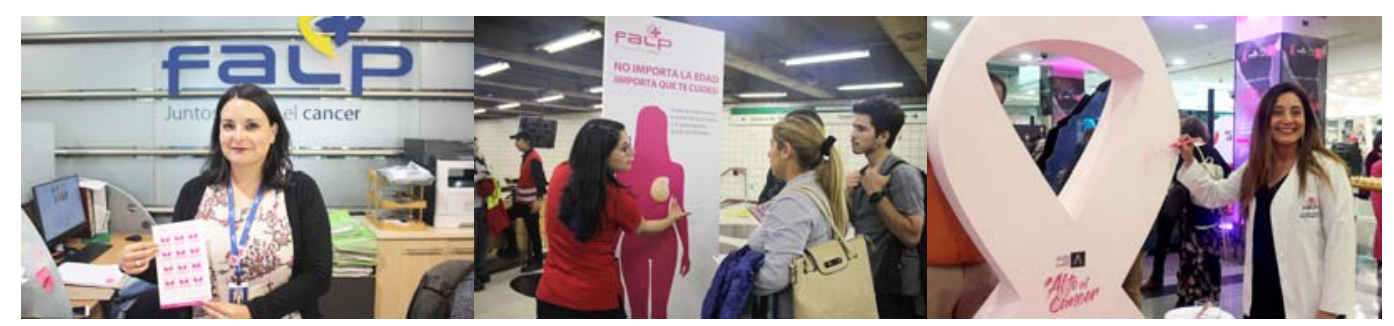

Hall Falp

Metro Baquedano

Alto Las Condes

Tótems informativos con aliados estratégicos. Recuperado de: https://www.facebook.com/Falpcomunicaciones/

\section{Hallazgos}

Mediante la recolección de información realizada con la Gerencia de Marketing y Comunicaciones de la institución, se establece que la FALP realiza una serie de acciones dentro de la campaña, las cuales se comienzan a planear entre los meses de abril y mayo, y que tienen año a año un mensaje y enfoque distinto. Siendo la maratón en conjunto con la empresa Avon, la acción más renombrada de la campaña contra el cáncer de mama. 
Sin embargo, el contacto que se tiene con los públicos objetivos para establecer las temáticas y construir la campaña no es suficiente. Actualmente, la FALP realiza este contacto con base a la experiencia y el contacto que profesionales de la salud tienen con los pacientes, de allí nace el slogan y acciones, las que son alineadas con el quehacer de la institución.

Por otra parte, se encuentra que la FALP aplica la campaña por diferentes medios tanto offline y online. En los medios online dispone del uso de RRSS con un amplio número de seguidores, lo que favorece la construcción de una comunidad virtual entorno a la marca, también cuenta con una página web institucional consolidada que sirve de plataforma para interactuar con los públicos objetivos.

La campaña de comunicación de lucha contra el cáncer de mama desarrollado por la FALP en octubre de 2018, en términos generales está diseñada de manera adecuada y con los parámetros propios de la comunicación estratégica.

\section{Conclusión}

Se realizó un análisis a la estrategia y plan de comunicación de la campaña comunicacional de lucha contra el cáncer de mama desarrollada por la FALP en octubre de 2018. El análisis se desarrolló con base a una investigación que permitió determinar como la FALP despliega su campaña, aplicando modelos de análisis del plan y campaña de comunicación que permitieron concluir que el desarrollo de la campaña de FALP es planificada de manera adecuada y está articulada a la estrategia de comunicación de la institución, puesto que esta trabaja en ser el referente más significativo en la lucha contra el cáncer en el país.

Mediante la aplicación de técnicas de recolección de información se pudo concluir que las principales características de la campaña para lograr concientizar a la población es la persuasión a las mujeres a realizarse el autoexamen, gracias a las alianzas que tiene la campaña, los medios de comunicación cumplen un rol importante para lograr informar a la comunidad sobre el objetivo de la misma.

El análisis del panel de expertos entrevistados por los investigadores, permite concluir que, pese a que la campaña de FALP cumple con los rasgos generales y principales, no cuenta con un proceso de medición y evaluación que permita determinar el impacto de esta en la población. Los expertos coincidieron en afirmar que la evaluación es de gran importancia en el éxito de una campaña y de la estrategia comunicacional de una institución, y sugieren utilizar los grupos focales como un 
método de evaluación de impacto muy efectivo en campañas de concientización como la desarrollada por la FALP.

Se debe realizar un proceso diagnóstico mucho más amplio que tenga en cuenta a un mayor número de actores de la campaña, que permita establecer un mejor enfoque de las acciones que componen la misma. Los expertos señalan que es necesario conocer mejor la audiencia previa al diseño de la campaña para garantizar esta con temáticas aplicadas a las necesidades propias de cada público. En ese sentido, siendo la FALP una institución renombrada en cuanto al tratamiento del cáncer en todo el país, la campaña contra el cáncer de mama realiza pocas acciones en las regiones, siendo esta una falencia que, en las entrevistas desarrolladas, reconoce y tiene en cuenta como un aspecto a mejorar su gerencia de comunicaciones y marketing.

En medios offline, la FALP cuenta con importantes alianzas que permiten comunicar su campaña por medio de revistas, televisión, tótems informativos, folletería y unidades móviles que se desplazan a diferentes comunas en gran parte del país. Sin embargo, sería recomendable para el equipo de comunicaciones y marketing de la institución, que el manejo de las redes sociales y en general, su política de marketing digital sea articulada a la estrategia de la campaña, logrando no solamente un alcance orgánico, para así lograr potenciar y que esta logre tener mayor alcance. Se recomienda realizar una campaña digital paga que el alcance de la misma y de este modo hacer un mayor aprovechamiento de las diferentes plataformas digitales utilizadas por la FALP.

Lo anterior permite determinar que parte de la hipótesis no se cumple, porque la FALP no establece ni aplica un modelo de mediciones efectivo al momento de implementar la campaña comunicacional. Esta falencia afecta la campaña y no aporta una retroalimentación adecuada que permite conocer la opinión de los públicos objetivos para mejorar las acciones que componen la estrategia y de esta manera aumentar su impacto social. Es por esto, que se recomienda la implementación de nuevas herramientas de evaluación que puedan potenciar la campaña contra el cáncer de mama y ayuden a mejorar sus resultados y alcances.

Para poder mejorar la campaña se debe establecer un modelo de medición y evaluación que esté integrado por los KPIs necesarios para identificar el nivel de impacto y aceptación de las acciones que componen la campaña. Con el proceso de medición y evaluación la FALP lograría saber de qué manera se está concientizando a los públicos objetivos y poder reorientar las acciones necesarias para mejorar la campaña y aumentar su nivel de impacto. 
Finalmente, en el desarrollo del presente proyecto de investigación se notó que en Chile existen pocos antecedentes de investigación que analice las campañas comunicacionales que buscan concientizar la lucha contra el cáncer de mama, lo que sirve de precedente para que otros investigadores tengan un punto de partida que ayuden a realizar estudios, los cuales permitan aportar a mejorar este tipo de campañas. Además, la investigación es un referente para futuros estudios desde el área de comunicación estratégica aplicados a campañas de concientización en temas de salud.

\section{Referencias}

Águeda, E. T. (2008). Principios del Marketing. Madrid: ESIC.

Alard, J. (2017). Campaña de Comunicación Integral 360. Rethink by Esic Business \& Marketing School. Recuperado de https://www.esic.edu/rethink/2017/10/26/campana-de-comunicacion-integral$360 /$

Alcalay, R. (1992). La comunicación para la salud como disciplina en las universidades estadounidenses. Revista Panamericana de Salud Pública, 5, 192-195.

Arcila, M. (2012). Comunicación Estratégica, y estrategias de comunicación para el desarrollo de las organizaciones. Pereira: Universidad Católica de Pereira.

Arribas, A., Herrera Echeñique, R. \& Pérez, R. A. (2017). Nueva Teoría Estratégica: Repensando la estrategia desde la comunicación. Razón y Palabra.

Balcázar, P. (2005). Investigación Cualitativa. México: Universidad Autónoma del Estado de México.

Cerullo, R., \& Weisenfeld, E. (2005). La concientización en el trabajo psicosocial comunitario desde la perspectiva de sus actores. Revista de Psicología, X(2).

Coimbra, M. \& Teixeira, L. (2010). As campanhas educativas contra o câncer. História, Ciências, Saúde - Manguinhos, 17(1) 223-241.

Costa, J. (2012). Construcción y gestión estratégica de la marca: Modelo MasterBrand. Revista Luciérnaga-Comunicación, 4(8), 20-25.

Chile. Ministerio de Salud (2017). Día contra el cáncer de mama. Recuperado de https://www.minsal.cl/dia-contra-el-cancer-mama/

Delarbre, R. T. (27 de Febrero de 2014). El sitio de Raúl Trejo Delarbre. Recuperado de http://raultrejo.tripod.com/Mediosensayos/medios.htm 
Fraenkel, J. R., Wallen, N. E., \& Hyun, H. H. (2011). How to design and evaluate research in education. Nueva York: McGraw-Hill Humanities/Social Sciences/Languages.

Freire, P. (1973). La desmitificación de la concientización. Cuernavaca: Centro Intercultural de Documentación.

Fundación Arturo López Pérez (2017). Informe de Memoria de Gestión FALP. Santiago.

Garrido, F. J. (2004). Comunicación estratégica: Las claves de la comunicación empresarial en el siglo XXI. España: Ediciones Gestión 2000.

Godoy, M. E. (2016). El brief como elemento esencial de toda gestión publicitaria. Contribuciones a las Ciencias Sociales, (2016-06).

Icaza, G., Núñez, L., \& Bugueño, H. (2017). Descripción epidemiológica de la mortalidad por cáncer de mama en mujeres en Chile. Revista Médica de Chile, 145(1), 106-114. doi: 10.4067/S0034-98872017000100014

Martins, A., Barbosa, T., \& Cezar, L. (2019). Análise da campanha Outubro Rosa de prevenção do câncer de mama em Viçosa, MG. Revista de Ciências Humanas, 12(2). Recuperado de https://periodicos.ufv.br/RCH/article/view/3936

Porroche-Escudero, A. (2016). Empoderamiento: el santo grial de las campañas de cáncer de mama. Revista Internacional de Sociología, 74(2), 031. doi: 10.3989/ris.2016.74.2.031

Porter, M. E. (2015). Estrategia competitiva: Técnicas para el análisis de los sectores industriales y de la competencia. México: Grupo Editorial Patria.

Prolla, C. M. D., Silva, P. S. D., Netto, C. B. O., Goldim, J. R., \& Ashton-Prolla, P. (2015). Knowledge about breast cancer and hereditary breast cancer among nurses in a public hospital. Revista latino-americana de enfermagem, 23(1), 90-97. doi: 10.1590/0104-1169.0185.2529

Renau, M. P., \& Font, L. L. (2017). Merco y RepTrak Pulse: Comparación cualitativa de atributos, variables y públicos. Revista ICONO14 Revista científica de Comunicación y Tecnologías emergentes, 15(2), 190-219. doi: 10.7195/ri14.v15i2.1077

Rivera, O. (1991). Los Conceptos de Misión, Visión y Propósito Estratégico. España: Universidad de Deusto, España.

Rogers, E. M. (1996). Up-to-date report. Journal of Health communication, 1(1), 15-24.

Scheinsohn, D. (2008). Comunicación estratégica. Cuaderno 28, 28, 145-150. 
The Economist Intelligence Unit. (2017). Control del cáncer, acceso y desigualdad en América Latina: Una historia de luces y sombras. Londres. Recuperado de: https://eiuperspectives.economist.com/sites/default/files/images/Cancer_cont rol_access_and_inequality_in_Latin_America_SPANISH.pdf

Tur-Viñes, V., \& Monserrat-Gauchi, J. (2014). El plan estratégico de comunicación. Estructura y funciones. Razón y palabra, 18(88).

U.S. Department of Health and Human Services. (2012). Healthy People 2010 Final Review. Oms, Hyattsville.

Van der Hofstadt, C. J. (2005). El libro de las Habilidades de la Comunicación. España: Ediciones Díaz de Santos S.A.

\begin{abstract}
Guillermo Antonio Gamboa Peña
Comunicador Social, Universidad de Pamplona, Colombia. Diplomado en Estrategia y Comunicación Digital y Diplomado en Gestión de Crisis y Resolución de Conflicto, Universidad de las Artes, Ciencias y Comunicación, UNIACC - Chile. Magíster en Gestión Estratégica de la Comunicación, UNIACC - Chile. Director de comunicaciones de Spirulina mater y Director de comunicación y marketing de la empresa Campos de Chile.

Email: guillermogamboa99@hotmail.com

\section{Valeska Fajardo Henríquez}

Periodista y Relacionadora Pública, Universidad Santo Tomás, Chile. Diplomada en Estrategia y Comunicación Digital y Diplomada en Gestión de Crisis y Resolución de Conflicto, Universidad de las Artes, Ciencias y Comunicación, UNIACC - Chile. Magíster en Gestión Estratégica de la Comunicación, UNIACC - Chile.

Email: valeska.fajardo.h@gmail.com

\section{Cielo Lengua Buelvas}

Comunicadora social y periodista, Universidad de Pamplona, Colombia. Diplomado en Educomunicación, Universidad de Pamplona. Diplomado en Estrategia y Comunicación Digital y Diplomado en Gestión de Crisis y Resolución de Conflicto, Universidad de las Artes, Ciencias y Comunicación, UNIACC - Chile. Magíster en Gestión Estratégica de la Comunicación, UNIACC - Chile.

Email: cilebu@gmail.com
\end{abstract}

